\title{
Further optimizing memory assessment tests: considering the neglected influencing factors
}

Volume 2 Issue 6 - 2015

\section{Editorial}

Memory assessment is an important part of divers studies in different fields. ${ }^{1-4}$ Indeed, many diseases such as neurodegenerative diseases including Alzheimer's disease, testing selected drugs or methodologies require assessment memory-related parameters. Up to now, a number of methods is used in experimental pharmacology/ toxicology and physiology, including Y-maze,,${ }^{5-7}$ Morris water maze, ${ }^{8-}$ ${ }^{10}$ functional magnetic resonance imaging, ${ }^{11}$ to explore different aspects and types of memories in divers context and at different levels.

However, the divers used methods have been described with some limits therefore a need to further optimize and validate the methods toward more accurate results. Herein, we would like to introduce divers several points that can be taken into consideration during the validation/ optimization process. Since not very much is known about the memory process and about the factors that can influence it is highly recommended to avoid or minimize the factor that could have an influence on the memory performance even the elements that might an indirect influence especially when conducting memory assessment tests. This could include light intensity, air pressure, olfactory stimuli, stress and noise.

In addition to such approaches, conducting the same test on similar control groups under similar condition at the same locations and during the same time frame remains also a necessity. Furthermore, it would further improve the memory test optimization if parameters related to memory performance such as selected neurotransmitters are also measures during the memory assessment. Importantly, considering the physiological factors such as brain regions maturity, locomotors, visibility and age is also a key elements while interpreting the tests results and therefore are also to be considered within the optimization process.

\section{Acknowledgements}

Abdelaziz Ghanemi is a recipient of a 2013 CAS-TWAS President's Postgraduate Fellowship.

\section{Conflicts of Interest}

The author declares no conflict of interest.

\section{References}

1. Ivanova MV, Hallowell B. A new modified listening span task to enhance validity of working memory assessment for people with and without aphasia. Journal of Communication Disorders. 2014;52:78-98.

2. O'Bryant SE, Duff K, Fisher J, et al. Performance profiles and cut-off scores on the Memory Assessment Scales. Arch Clin Neuropsychol. 2004;19(4):489-496.

3. Injoque-Ricle I, Calero $\mathrm{AD}$, Alloway $\mathrm{TP}$, et al. Assessing working memory in Spanish-speaking children: Automated Working Memory Assessment battery adaptation. Learning and Individual Differences. 2011;21(1):78-84.

\author{
Abdelaziz Ghanemi ${ }^{1,2,3}$ \\ 'Key Laboratory of Animal Models and Human Disease, \\ Mechanisms of Chinese Academy of Sciences \& Yunnan Province, \\ Kunming Institute of Zoology, China \\ ${ }^{2}$ Kunming College of Life Science, University of Chinese \\ Academy of Sciences, China \\ ${ }^{3}$ University of Chinese Academy of Sciences, China
}

Correspondence: Abdelaziz Ghanemi, Key Laboratory of Animal Models and Human Disease Mechanisms, Kunming Institute of Zoology Chinese Academy of Sciences, No.32 Jiaochang Donglu, Kunming 650223, Yunnan Province, China, Tel 0086-15887090734, Email ghanemiabdelaziz@hotmail.com

Received: January 0I, 1970 | Published: September 08, 2015

4. Catale C, Meulemans T, Kerrouche B, et al. Everyday memory assessment following childhood acquired brain injury (ABI). Annals of Physical and Rehabilitation Medicine. 2014;57(Suppl 1):e341.

5. Dridi I, Leroy D, Guignard C, et al. Dietary early-life exposure to contaminated eels does not impair spatial cognitive performances in adult offspring mice as assessed in the Y-maze and the Morris water maze. Nutr Res. 2014;34(12):1075-1084.

6. Cai ZL, Wang CY, Jiang ZJ, et al. Effects of cordycepin on Y-maze learning task in mice. Eur J Pharmacol. 2013;714(1-3):249-253.

7. Aoki R, Tsuboi T, Okamoto H. Y-maze avoidance: An automated and rapid associative learning paradigm in zebrafish. Neurosci Res. 2015;91:69-72.

8. Kulikov AV, Fursenko DV, Khotskin NV, et al. Spatial learning in the Morris water maze in mice genetically different in the predisposition to catalepsy: The effect of intraventricular treatment with brain-derived neurotrophic factor. Pharmacol Biochem Behav. 2014;122:266-272.

9. Péczely L, Ollmann T, László K, et al. Effects of ventral pallidal D1 dopamine receptor activation on memory consolidation in morris water maze test. Behav Brain Res. 2014;274:211-218.

10. Daniels BP, Sestito SR, Rouse ST. An expanded task battery in the Morris water maze reveals effects of Toxoplasma gondii infection on learning and memory in rats. Parasitol Int. 2015;64(1):5-12.

11. McAndrews MP. Memory assessment in the clinical context using functional magnetic resonance imaging: a critical look at the state of the field. Neuroimaging Clin N Am. 2014;24(4):585-597. 